SLAC-PUB-10781

BABAR-PROC-04/112

October, 2004

\title{
Charm decays at BABAR
}

\author{
M. Charles \\ University of Iowa \\ SLAC M/S 35, 2575 Sand Hill Road, Menlo Park, CA 94025 \\ (representing the BABAR Collaboration)
}

\begin{abstract}
The results of several studies of charmed mesons and baryons at BABAR are presented. First, searches for the rare decays $D^{0} \rightarrow l^{+} l^{-}$are presented and new upper limits on these processes are established. Second, a measurement of the branching fraction of the isospin-violating hadronic decay $D_{s}^{*}(2112)^{+} \rightarrow D_{s}^{+} \pi^{0}$ relative to the radiative decay $D_{s}^{*}(2112)^{+} \rightarrow D_{s}^{+} \gamma$ is made. Third, the decays of $D_{s J}^{*}(2317)^{+}$and $D_{s J}(2460)^{+}$mesons are studied and ratios of branching fractions are measured. Fourth, Cabibbo-suppressed decays of the $\Lambda_{c}^{+}$are examined and their branching fractions measured relative to Cabibbo-allowed modes. Fifth, the $\Xi_{c}^{0}$ is studied through its decays to $\Xi^{-} \pi^{+}$and $\Omega^{-} K^{+}$; in addition to measuring the ratio of branching fractions for $\Xi_{c}^{0}$ produced from the $c \bar{c}$ continuum, the uncorrected momentum spectrum is measured, providing clear confirmation of $\Xi_{c}^{0}$ production in $B$ decays.
\end{abstract}

Contributed to the Proceedings of the $32^{\text {nd }}$ International Conference on High Energy Physics, 8/16/2004-8/22/2004, Beijing, China

Stanford Linear Accelerator Center, Stanford University, Stanford, CA 94309

Work supported in part by Department of Energy contract DE-AC02-76SF00515. 
Table 1: The expected background, the number of observed events, the corresponding branching fraction upper limit at the $90 \%$ confidence level (preliminary), and the previous upper limit for each decay mode.

\begin{tabular}{|l|ccc|}
\hline & $D^{0} \rightarrow e^{+} e^{-}$ & $D^{0} \rightarrow \mu^{+} \mu^{-}$ & $D^{0} \rightarrow e^{ \pm} \mu^{\mp}$ \\
\hline Expected $D^{0} \rightarrow h^{+} h^{-}$backgrnd. $(h=\pi, K)$ & 0.02 & $3.3 \pm 0.3$ & 0.21 \\
Expected combinatoric backgrnd. & $2.2 \pm 0.4$ & $1.3 \pm 0.3$ & $1.9 \pm 0.4$ \\
Candidates observed in signal window & 3 & 1 & 0 \\
90\% confidence level upper limit $\left(10^{-6}\right)$ & 1.2 & 1.3 & 0.81 \\
Previous upper limit $\left(10^{-6}\right)$ & 6.2 & 2.5 & 8.1 \\
\hline
\end{tabular}

Table 2: Preliminary ratios of branching fractions in the $D_{s J}$ system.

\begin{tabular}{|ll|}
\hline $\mathcal{B}\left(D_{s J}^{*}(2317)^{+} \rightarrow D_{s}^{+} \gamma\right) / \mathcal{B}\left(D_{s J}^{*}(2317)^{+} \rightarrow D_{s}^{+} \pi^{0}\right)$ & $<0.17 @ 95 \%$ C.L. \\
$\mathcal{B}\left(D_{s J}^{*}(2317)^{+} \rightarrow D_{s}^{+} \pi^{+} \pi^{-}\right) / \mathcal{B}\left(D_{s J}^{*}(2317)^{+} \rightarrow D_{s}^{+} \pi^{0}\right)$ & $<0.11 @ 95 \%$ C.L. \\
\hline $\mathcal{B}\left(D_{s J}(2460)^{+} \rightarrow D_{s}^{+} \gamma\right) / \mathcal{B}\left(D_{s J}(2460)^{+} \rightarrow D_{s}^{+} \pi^{0} \gamma\right)$ & $0.375 \pm 0.054 \pm 0.057$ \\
$\mathcal{B}\left(D_{s J}(2460)^{+} \rightarrow D_{s}^{+} \pi^{+} \pi^{-}\right) / \mathcal{B}\left(D_{s J}(2460)^{+} \rightarrow D_{s}^{+} \pi^{0} \gamma\right)$ & $0.082 \pm 0.018 \pm 0.011$ \\
$\mathcal{B}\left(D_{s J}(2460)^{+} \rightarrow D_{s}^{+} \pi^{0}\right) / \mathcal{B}\left(D_{s J}(2460)^{+} \rightarrow D_{s}^{+} \pi^{0} \gamma\right)$ & $<0.002 @ 95 \%$ C.L. \\
$\mathcal{B}\left(D_{s J}(2460)^{+} \rightarrow D_{s J}^{*}(2317)^{+} \gamma\right) / \mathcal{B}\left(D_{s J}(2460)^{+} \rightarrow D_{s}^{+} \pi^{0} \gamma\right)$ & $<0.23 @ 95 \%$ C.L. \\
\hline
\end{tabular}

\section{Introduction}

The data for these analyses are collected with the BABAR detector[1] at the PEP-II asymmetric $e^{+} e^{-}$ collider, operating at the $\Upsilon(4 S)$ resonance and at a center-of-mass $(\mathrm{CM})$ energy $\sim 40 \mathrm{MeV}$ below it. At these energies there is copious production of $c \bar{c}$ pairs from the continuum[2]. Combined with high integrated luminosity, this makes $B A B A R$ an excellent laboratory for studying charm production and decays. Detailed discussions of the topics in this paper may be found in the conference submissions $[3,4,5,6,7]$. All results are preliminary.

\section{Search for $D^{0} \rightarrow l^{+} l^{-}$}

In the Standard Model (SM), the decay modes ${ }^{1} D^{0} \rightarrow e^{+} e^{-}$and $D^{0} \rightarrow \mu^{+} \mu^{-}$are strongly suppressed by the GIM mechanism[8]; their branching fractions are estimated to be $10^{-23}$ and $3 \times 10^{-13}$ respectively[9]. The lepton flavour violating decay $D^{0} \rightarrow e^{ \pm} \mu^{\mp}$ is strictly forbidden in the SM. However, new physics could enhance these rates[9]. The results of searches for all three modes using $122 \mathrm{fb}^{-1}$ of data are presented in this paper.

The event selection comprises invariant mass cuts and particle identification (PID) criteria. In order to improve the purity of the $D^{0}$ candidate sample, it is required that an additional $\pi^{+}$track be present and that the $D^{0}$ candidate be consistent with a $D^{*+} \rightarrow D^{0} \pi^{+}$decay. The expected backgrounds and numbers of observed events are shown in Table 1, along with the preliminary upper limits[10]. The limits are normalized to the kinematically similar $D^{0} \rightarrow \pi^{+} \pi^{-}$mode[11]. These

\footnotetext{
${ }^{1}$ Unless otherwise stated, the use of charge conjugate modes is implied throughout.
} 

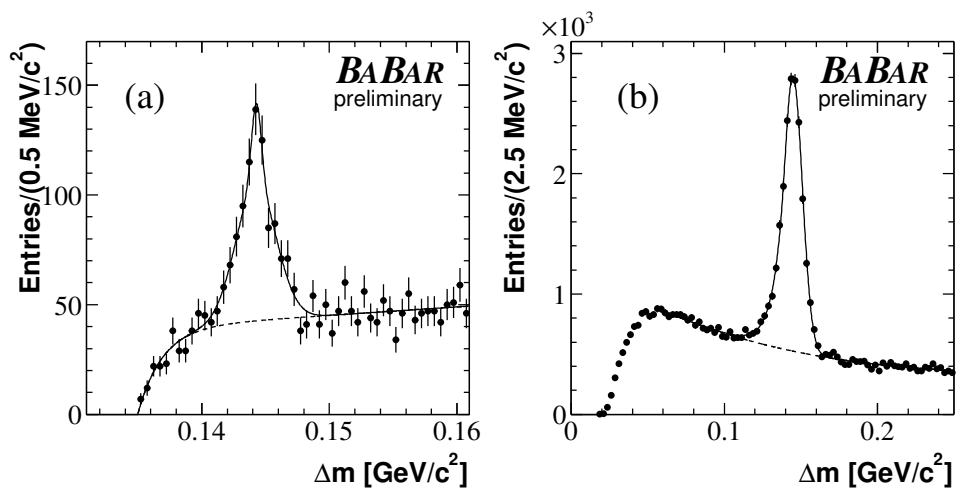

Figure 1: $\quad D_{s}^{*}(2112)^{+}$signals: (a) $m\left(K^{+} K^{-} \pi^{+} \pi^{0}\right)-m\left(K^{+} K^{-} \pi^{+}\right)$; (b) $m\left(K^{+} K^{-} \pi^{+} \gamma\right)-$ $m\left(K^{+} K^{-} \pi^{+}\right)$. The dots represent data points. The solid line shows the fitted function, and the dashed line indicates estimated background contribution.

represent significant improvements on the previous limits[12, 13], and further restrict possible new physics contributions to these decay processes.

\section{$3 \quad$ Measurement of $\mathcal{B}\left(D_{s}^{*}(2112) \rightarrow D_{s}^{+} \pi^{0}\right) / \mathcal{B}\left(D_{s}^{*}(2112) \rightarrow D_{s}^{+} \gamma\right)$}

Compared to $D_{s}^{*}(2112)^{+} \rightarrow D_{s}^{+} \gamma$, the hadronic decay $D_{s}^{*}(2112)^{+} \rightarrow D_{s}^{+} \pi^{0}$ is expected to be isospinsuppressed. Using chiral perturbation theory and assuming virtual $\eta$ emission, sizeable hadronic decay rates have been predicted[14], and were subsequently measured by the CLEO collaboration[15]. In this section, a BABAR measurement of the ratio using $90.4 \mathrm{fb}^{-1}$ of data is presented.

The $D_{s}^{+}$mesons are reconstructed using the decay $D_{s}^{+} \rightarrow \phi \pi^{+}, \phi \rightarrow K^{+} K^{-}$. PID requirements are imposed upon the kaons. The $D_{s}^{+}$candidate is then combined with a $\gamma$ or $\pi^{0}$ which satisfies selection criteria including mass, energy, and (for $\pi^{0}$ ) helicity angle requirements to form a $D_{s}^{*}(2112)^{+}$candidate. The mass offset spectra are shown in Figure 1. After correcting for efficiency and taking into account systematic uncertainties (predominantly due to momentum dependence of the efficiencies and limited statistics of the Monte Carlo simulation), the preliminary ratio of branching fractions is measured to $b^{2} 0.0621 \pm 0.0049 \pm 0.0063$. As a crosscheck, the non-isospinsuppressed decay $D^{* 0} \rightarrow D^{0} \pi^{0}$ relative to $D^{* 0} \rightarrow D^{0} \gamma$ was also measured. This was found to be $1.740 \pm 0.020 \pm 0.125$, consistent with the existing world average[16].

\section{$4 \quad D_{s J}^{+}$decays}

The unexpected discovery of the $D_{s J}^{*}(2317)^{+}$and $D_{s J}(2460)^{+}$mesons $[17,18]$ and subsequent studies of their decays $[19,20,21]$ have re-awoken interest in charm meson spectroscopy. In this section, new $B A B A R$ measurements of ratios of their branching fractions are presented. These measurements are made with inclusive $D_{s J}$ samples in $125 \mathrm{fb}^{-1}$ of data. In each case, $D_{s J}$ candidates are required to have a CM momentum $\left(p^{*}\right)$ of at least $3.2 \mathrm{GeV} / c$. This suppresses $D_{s J}$ production from $B$ decays. The preliminary ratios of branching fractions and upper limits obtained are shown in

\footnotetext{
${ }^{2}$ When a value is quoted with two uncertainties in this paper, the first is statistical and the second is systematic. When an upper limit is quoted, the limit incorporates the effects of both the statistical and the systematic uncertainty.
} 
Table 3: Preliminary $\Lambda_{c}^{+}$branching fraction ratios.

\begin{tabular}{|ll|}
\hline$\frac{\mathcal{B}\left(\Lambda_{c}^{+} \rightarrow \Lambda K^{+}\right)}{\mathcal{B}\left(\Lambda_{c}^{+} \rightarrow \Lambda \pi^{+}\right)}$ & $0.044 \pm 0.004 \pm 0.002$ \\
$\frac{\mathcal{B}\left(\Lambda_{c}^{+} \rightarrow \Sigma^{0} K^{+}\right)}{\mathcal{B}\left(\Lambda_{c}^{+} \rightarrow \Sigma^{0} \pi^{+}\right)}$ & $0.040 \pm 0.005 \pm 0.004$ \\
$\frac{\mathcal{B}\left(\Lambda_{c}^{+} \rightarrow \Lambda K^{+} \pi^{+} \pi^{-}\right)}{\mathcal{B}\left(\Lambda_{c}^{+} \rightarrow \Lambda \pi^{+}\right)}$ & $0.266 \pm 0.027 \pm 0.032$ \\
$\frac{\mathcal{B}\left(\Lambda_{c}^{+} \rightarrow \Sigma^{0} K^{+} \pi^{+} \pi^{-}\right)}{\mathcal{B}\left(\Lambda_{c}^{+} \rightarrow \Sigma^{0} \pi^{+}\right)}$ & $<0.039 @ 90 \%$ C.L. \\
\hline
\end{tabular}

Table 2. Details of the fitting procedure, including proper handling of reflections, are given in the conference submission[5]. In addition, the $D_{s}^{+} \pi^{ \pm}$invariant mass spectra were examined: no evidence of any narrow structure is found close to the $D_{s J}^{*}(2317)$ mass. This is consistent with the $D_{s J}^{*}(2317)$ being an isosinglet state.

\section{Cabibbo-suppressed $\Lambda_{c}^{+}$decays}

In this section, four new measurements of Cabibbo-suppressed $\Lambda_{c}^{+}$decays using $125 \mathrm{fb}^{-1}$ of data are presented. The decay products include a $\Lambda\left(p \pi^{-}\right)$or $\Sigma^{0}(\Lambda \gamma)$ plus one or more charged pions or kaons. Background is rejected through mass and flight distance cuts on the hyperons, PID requirements, an energy threshold for $\gamma$ candidates, and $p^{*}$ thresholds for $\Lambda_{c}^{+}$candidates. In each case, the branching fraction is measured relative to a similar, Cabibbo-allowed mode. The preliminary results, corrected for efficiency, are given in Table 3. These represent significant improvements over existing results[22]. This is the first observation of the decay $\Lambda_{c}^{+} \rightarrow \Lambda K^{+} \pi^{+} \pi^{-}$; the mass spectrum for this mode is shown in Figure 2 (a). The decay mode $\Lambda_{c}^{+} \rightarrow \Sigma^{0} K^{+} \pi^{+} \pi^{-}$appears to be strongly suppressed relative to $\Lambda_{c}^{+} \rightarrow \Sigma^{0} K^{+}$(Figure 2); at present there is no explanation of this intriguing feature.

\section{$6 \quad \Xi_{c}^{0}$ production and decays}

In this section, the ratio of $\Xi_{c}^{0}$ branching fractions to the $\Omega^{-} K^{+}$and $\Xi^{-} \pi^{+}$final states is measured using $116 \mathrm{fb}^{-1}$ of data. In addition, the production of $\Xi_{c}^{0}$ in $B$ decays is observed. Although copious production of $\Xi_{c}^{0}$ and $\Xi_{c}^{+}$in $B$ decays has been predicted[23], this process has been observed previously only by CLEO, with a significance of $\sim 3 \sigma$ in the $\Xi_{c}^{0} \rightarrow \Xi^{-} \pi^{+}$decay mode and $\sim 4 \sigma$ in a related $\Xi_{c}^{+}$decay mode[24].

$\Xi_{c}^{0}$ candidates are selected using invariant mass and flight distance criteria for the intermediate hyperons, plus PID requirements on kaon and proton tracks. The $p^{*}$ spectra shown in Figure 3 are obtained by subtracting background contributions, estimated using events from the mass sidebands, and are not corrected for efficiency. The peaks below $1.5 \mathrm{GeV} / c$ visible in the on-peak samples (Figures 3(c) and (d)) provide clear confirmation of $\Xi_{c}^{0}$ production in $B$ decays.

To measure the ratio of branching fractions to the two decay modes, the candidates are further required to have $p^{*}>1.8 \mathrm{GeV} / c$. After correcting for efficiency, the preliminary ratio of branching fractions is found to be $0.296 \pm 0.018 \pm 0.030$, where the dominant systematic uncertainties are from the fitting procedure and efficiency loss due to detector acceptance. This improves upon the previous measurement by CLEO[25] and is consistent with a spectator quark model prediction[26]. 

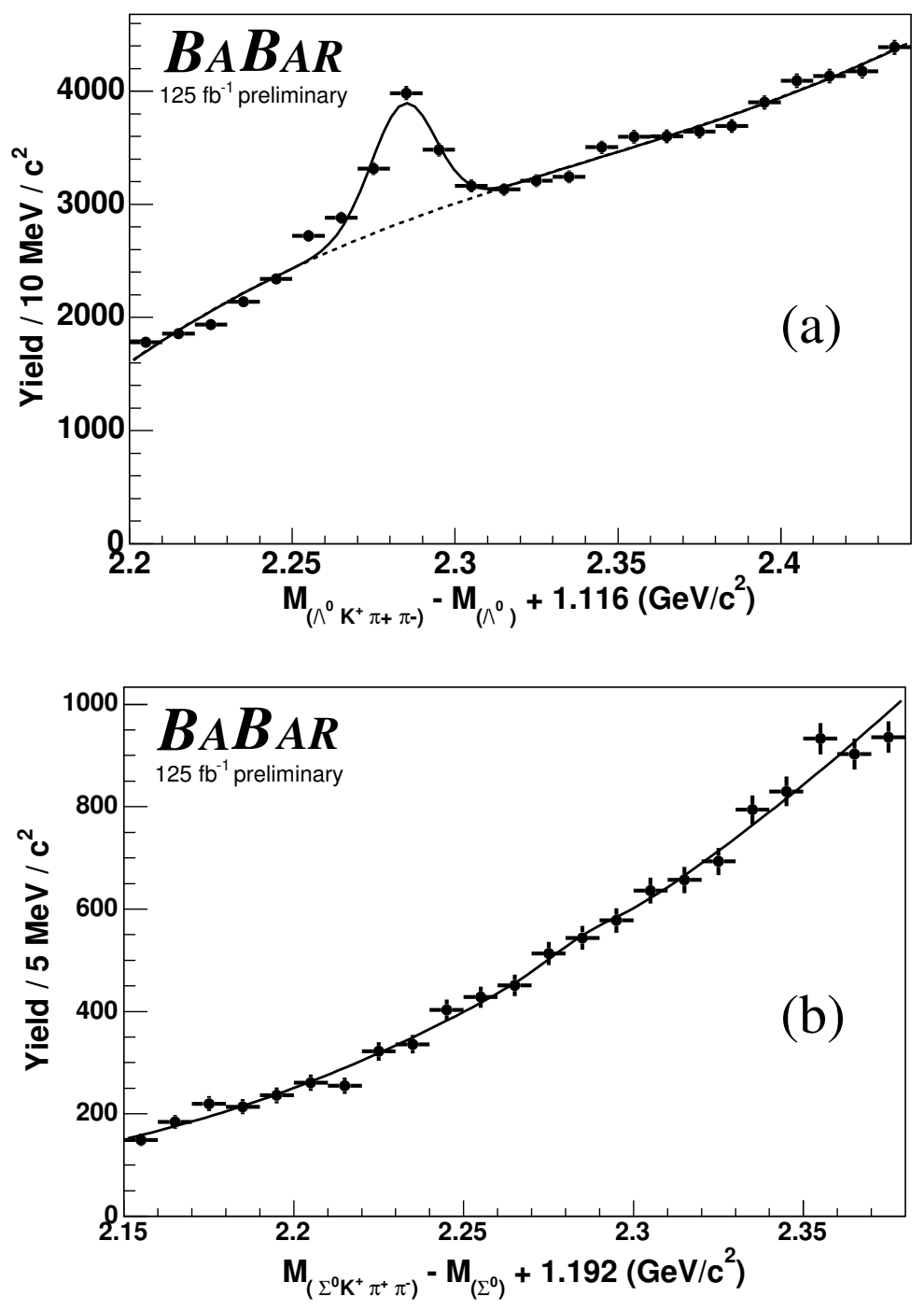

Figure 2: The invariant mass spectra for (a) $\Lambda K^{+} \pi^{+} \pi^{-}$, (b) $\Sigma^{0} K^{+} \pi^{+} \pi^{-}$. A clear $\Lambda_{c}^{+}$peak can be seen in (a), but not in (b). 

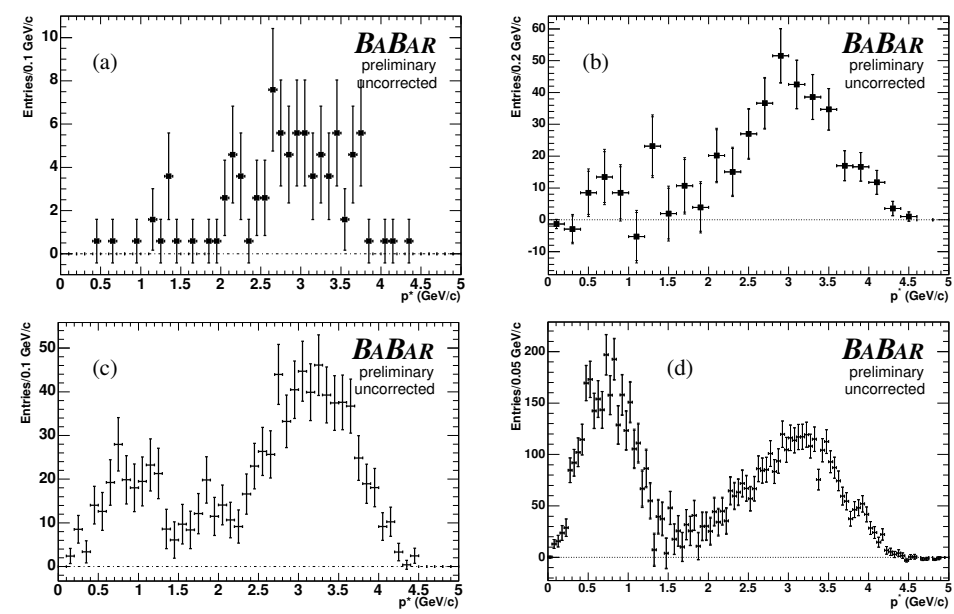

Figure 3: Uncorrected $p^{*}$ distributions. The $\Xi_{c}^{0} \rightarrow \Omega^{-} K^{+}$spectra are shown in (a) and (c), and the $\Xi_{c}^{0} \rightarrow \Xi^{-} \pi^{+}$spectra are shown in (b) and (d). Figures (a) and (b) correspond to $11 \mathrm{fb}^{-1}$ of data taken at $\mathrm{CM}$ energy $\sim 40 \mathrm{MeV}$ below the $\Upsilon(4 S)$, while (c) and (d) correspond to $104 \mathrm{fb}^{-1}$ of data taken at the $\Upsilon(4 S)$ resonance.

\section{Conclusions}

BABAR has a very active charm physics program. The topics discussed in this paper are only a fraction of those presented at the $32^{\text {nd }}$ International Conference on High Energy Physics at Beijing, and with the excellent luminosity achieved by PEP-II we expect many high-precision results to follow.

\section{References}

[1] The BABAR Collaboration, B. Aubert et al., Nucl. Instrum. Methods A479, 1-116 (2002).

[2] BABAR Collaboration, P. F. Harrison and H. R. Quinn, ed., "The BABAR Physics Book," SLAC-R-504 (1998).

[3] BABAR Collaboration, B. Aubert et al., ICHEP 2004 abstract 11-0964, hep-ex/0408023, accepted for publication in Phys. Rev. Lett..

[4] BABAR Collaboration, B. Aubert et al., ICHEP 2004 abstract 11-0953, hep-ex/0408094.

[5] BABAR Collaboration, B. Aubert et al., ICHEP 2004 abstract 10-0631, hep-ex/0408067.

[6] BABAR Collaboration, B. Aubert et al., ICHEP 2004 abstract 11-0963, hep-ex/0408024.

[7] BABAR Collaboration, B. Aubert et al., ICHEP 2004 abstract 11-0938, hep-ex/0408056.

[8] S. L. Glashow, J. Iliopoulos and L. Maiani, Phys. Rev. D 2, 1285 (1970).

[9] G. Burdman, E. Golowich, J. Hewett and S. Pakvasa, Phys. Rev. D 66, 014009 (2002). 
[10] J. Conrad, O. Botner, A. Hallgren and C. Perez de los Heros, Phys. Rev. D 67, 012002 (2003).

[11] K. Hagiwara et al. [Particle Data Group Collaboration], Phys. Rev. D 66, 010001 (2002).

[12] E791 Collaboration, E. M. Aitala et al., Phys. Lett. B 462, 401 (1999).

[13] HERA-B Collaboration, I. Abt et al., arXiv:hep-ex/0405059.

[14] P. L. Cho and M. B. Wise, Phys. Rev. D 49, 6228 (1994).

[15] CLEO Collaboration, J. Gronberg et al., Phys. Rev. Lett. 75, 3232 (1995).

[16] Particle Data Group, S. Eidelwan et al., Phys. Lett. B 592, 1 (2004).

[17] BABAR Collaboration, B. Aubert et al., Phys. Rev. Lett. 90, 242001 (2003).

[18] CLEO Collaboration, D. Besson et al., Phys. Rev. D68, 032002 (2003).

[19] Belle Collaboration, K. Abe et al., Phys. Rev. Lett. 92, 012002 (2004).

[20] Belle Collaboration, P. Krokovny et al., Phys. Rev. Lett. 91, 262002 (2003).

[21] BABAR Collaboration, B. Aubert et al., Phys. Rev. D69, 031101 (2004).

[22] Belle Collaboration, K. Abe. et al., Phys. Lett. B 524, 33 (2002).

[23] P. Ball and H.G. Dosch, Z. Phys. C 51, 455 (1991).

[24] CLEO Collaboration, B. Barish et al., Phys. Rev. Lett. 79, 3599 (1997).

[25] CLEO Collaboration, S. Henderson et al., Phys. Lett. B 283, 161 (1992).

[26] J. G. Korner and M. Kramer, Z. Phys. C 55, 659 (1992). 